\title{
Domain walls and their stability
}

\author{
Z. Jovanoski ${ }^{1}$ \\ I. N. Towers ${ }^{2}$ \\ N. A. Ansari ${ }^{3}$
}

(Received 15 August 2008; revised 21 September 2009)

\begin{abstract}
Using Jacobi elliptic functions we find a complete set of exact periodic and soliton solutions for two waves co-propagating in a defocusing nonlinear medium. We show using both linear stability analysis and numerical simulation that these solutions are stable.
\end{abstract}

\section{Contents}

1 Introduction

C1053

2 Jacobi elliptic function method

C1054

3 Exact periodic and solitary wave solutions

C1056

4 Linear stability analysis

C1060

5 Conclusion

C1062

References

\section{C1063}

http://anziamj.austms.org.au/ojs/index.php/ANZIAMJ/article/view/1468 gives this article, (c) Austral. Mathematical Soc. 2009. Published September 29, 2009. ISSN 1446-8735. (Print two pages per sheet of paper.) 


\section{Introduction}

The construction of exact solutions of nonlinear partial differential equations is one of the most important and essential tasks in nonlinear science. This pursuit led to the development of new mathematical techniques such as the inverse scattering transform, Bäcklund transformation, and Hirota's transformation [1]. These techniques are often employed on the nonlinear Schrödinger (NLS) equation which is often viewed as an archetype for many nonlinear evolution equations. The NLS equation is one of a number of nonlinear evolution equations for which exact soliton solutions are available, both bright and dark [2].

For the case of two electric fields of the form $\psi_{1}(z, x) e^{-i \omega t}$ and $\psi_{2}(z, x) e^{-i \omega t}$, where $\omega$ is the frequency, co-propagating in a third order nonlinear medium satisfy the coupled nonlinear Schrödinger wave equation [3]

$$
\begin{aligned}
& i \frac{\partial \psi_{1}}{\partial z}+\frac{\sigma_{1}}{2} \frac{\partial^{2} \psi_{1}}{\partial x^{2}}+\left(\left|\psi_{1}\right|^{2}+\alpha\left|\psi_{2}\right|^{2}\right) \psi_{1}=0, \\
& i \frac{\partial \psi_{2}}{\partial z}+\frac{\sigma_{2}}{2} \frac{\partial^{2} \psi_{2}}{\partial x^{2}}+\left(\left|\psi_{2}\right|^{2}+\alpha\left|\psi_{1}\right|^{2}\right) \psi_{2}=0,
\end{aligned}
$$

where $\psi_{i}$ are the complex envelopes of the polarisation components of the $i$ th wave, $z$ is the propagation axis, $x$ is the transverse spatial coordinate and $\sigma_{i}= \pm 1$. The independence of the field envelope on time means that group velocity dispersion does not enter the problem. The coupling constant, $\alpha$, also known as the cross-phase modulation, is

$$
\alpha=\frac{1+B}{1-B},
$$

where $\mathrm{B}=\chi_{1221}^{(3)} / \chi_{1111}^{(3)}$ and $\chi_{i j k l}^{(3)}$ is the third order non-linear susceptibility tensor. In the absence of coupling $(\alpha=0)$, the two waves propagate into the medium without interacting with each other. Kivshar and Agrawal [4] provide an extensive list of vector soliton solutions for the above system of equations according to the signs of $\sigma_{i}$. 
Apart from soliton solutions, these equations are also known to exhibit periodic solutions $[5,6]$. Of particular interest to us is the case $\sigma_{i}=-1$, which corresponds to the diffraction experienced by a two dimensional spatial beam in a self-defocusing non-linear medium [7]. Haelterman and Sheppard [7] employed a shooting method to numerically solve equations (1)-(2). They demonstrated the existence of stationary periodic solutions that consist of the superposition of two periodic waves of orthogonal polarisation that are $\pi$ radians out-of-phase. Solutions of different periods were numerically found for several values of $\alpha$. As the period was permitted to grow without bound, the periodic waves degenerated to dark solitons. No exact analytic solutions for the system were found by Haelterman and Sheppard [7]. Our aim is to look for exact analytic solutions of the system for which $\sigma_{i}=-1$. We look for solutions in terms of Jacobi elliptic functions. In part, this is motivated by the successful implementation of such functions to solve modified versions of the coupled NLS equation $[6,8]$ and to the self-focusing version of our system [9]. However, the articles cited here are merely indicative of what is available in the literature and are not intended to be exhaustive.

The mathematical framework for using the elliptic functions is laid out in Section 2 and the solutions are derived in Section 3. Here we find some well known solutions but also some new solutions that are totally distinct from any other solution set. These solutions constitute a new, rather simple, set of exact solutions that only exist under certain conditions. Section 4 studies their stability.

\section{Jacobi elliptic function method}

We define

$$
\psi_{1}=u(\eta) e^{i \beta z} \quad \text { and } \quad \psi_{2}=v(\eta) e^{i \beta z}
$$


where $\eta=k x$ and $k$ is the inverse of the beam width and substitute equations (3) into equations (1)-(2) to find

$$
\begin{aligned}
& \frac{k^{2}}{2} \frac{d^{2} u}{d \eta^{2}}+\beta u-u^{3}-\alpha v^{2} u=0 \\
& \frac{k^{2}}{2} \frac{d^{2} v}{d \eta^{2}}+\beta v-v^{3}-\alpha u^{2} v=0
\end{aligned}
$$

We write the solutions to the above system as a finite sum of powers of a Jacobi elliptic sine function

$$
\begin{aligned}
& u=\sum_{j=0}^{n_{1}} a_{j} \operatorname{sn}^{j}(\eta, m), \\
& v=\sum_{l=0}^{n_{2}} b_{l} \operatorname{sn}^{l}(\eta, m),
\end{aligned}
$$

where the constant parameter, $m \in[0,1]$, is the modulus of the elliptic function. We determine the values of $n_{1}$ and $n_{2}$ by balancing the highest order derivatives with the nonlinear contributions. In this case we get $n_{1}=$ $n_{2}=1$. Hence our solution takes on the simple form

$$
\begin{aligned}
& u=a_{0}+a_{1} \operatorname{sn}(\eta, m), \\
& v=b_{0}+b_{1} \operatorname{sn}(\eta, m) .
\end{aligned}
$$

The modulus $m$ has a significant impact on the shape of the solutions. For $\mathrm{m} \rightarrow 1$, we get the following ansatz

$$
\begin{aligned}
& u=a_{0}+a_{1} \tanh (\eta) \\
& v=b_{0}+b_{1} \tanh (\eta)
\end{aligned}
$$

so the soliton wave solution can be recovered from the periodic ones. 


\section{$3 \quad$ Exact periodic and solitary wave solutions}

The above equations represent the general solution of the steady state propagation of two coupled waves in a third order non-linear medium. In order to work out the coefficients $a_{0}, b_{0}, a_{1}$ and $b_{1}$ in equations (6)-(7), we substitute equations (6)-(7) into equations (4)-(5) and collect the coefficients of resulting powers of $\operatorname{sn}(\eta, m)$. This leads to a system of algebraic equations from which the coefficients were found using MATHEMATICA.

Several solution sets were found, some well known $(\alpha=1)$ and others new. New analytic solutions were found only in the case $\alpha=3$. For $\alpha=1$ equations (4)-(5) are known to be integrable via the inverse scattering transform. The solutions are solitons rather than merely solitary waves. The main distinction being that solitons undergo elastic collisions whereas solitary waves do not. In the case $\alpha=3$ equations (4)-(5) are also integrable. Recently, Jovanoski et al. [10] reported on periodic solutions for $\alpha=3$ in the restricted case $u \geqslant 0$ and $v \geqslant 0$, namely,

$$
\begin{aligned}
& u=\frac{\sqrt{\beta}}{2}\left[1+m \sqrt{\frac{2}{1+m^{2}}} \text { sn }\left(\sqrt{\frac{2 \beta}{1+m^{2}}} x, m\right)\right], \\
& v=\frac{\sqrt{\beta}}{2}\left[1-m \sqrt{\frac{2}{1+m^{2}}} \operatorname{sn}\left(\sqrt{\frac{2 \beta}{1+m^{2}}} x, m\right)\right],
\end{aligned}
$$

which translates to the dark soliton solution

$$
\begin{aligned}
& u=\frac{\sqrt{\beta}}{2}[1+\tanh (\sqrt{\beta} x)], \\
& v=\frac{\sqrt{\beta}}{2}[1-\tanh (\sqrt{\beta} x)] .
\end{aligned}
$$

Figure 1 shows set of solutions given by equations (8)-(9) and (10)-(11). The periodic solutions are $2 \mathrm{~K}(\mathrm{~m}) \sqrt{\left(1+\mathrm{m}^{2}\right) / 2 \beta}$ radians out-of-phase, where $\mathrm{K}(\mathrm{m})$ is the complete elliptic integral of the first kind. This is consistent with 

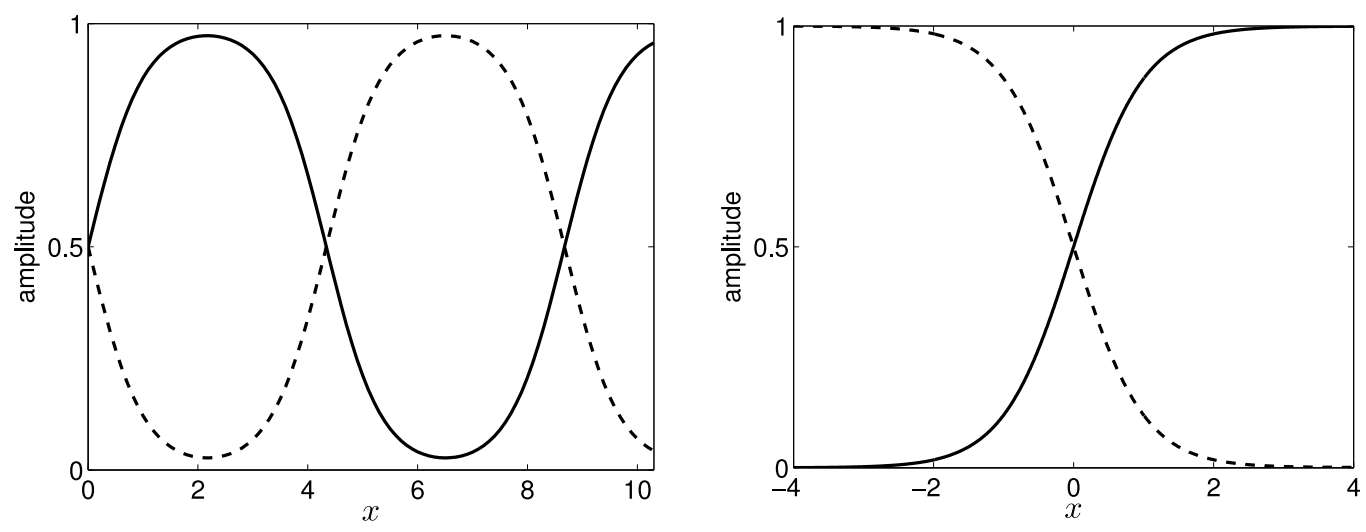

Figure 1: Exact out-of-phase solutions for $\beta=1$ and $\alpha=3$. To the left are the periodic solutions and to the right the soliton solutions (domain walls) with fields $u$ (solid line) and $v$.

the numerical simulations of Haelterman and Sheppard [7]. By relaxing the restriction on the signs of $u$ and $v$ means that $(-\mathfrak{u},-v)$ is another solution. The above system also supports an in-phase periodic solution

$$
\begin{aligned}
& u=\frac{\sqrt{\beta}}{2}\left[1+m \sqrt{\frac{2}{1+m^{2}}} \operatorname{sn}\left(\sqrt{\frac{2 \beta}{1+m^{2}}} x, m\right)\right], \\
& v=-\frac{\sqrt{\beta}}{2}\left[1-m \sqrt{\frac{2}{1+m^{2}}} \operatorname{sn}\left(\sqrt{\frac{2 \beta}{1+m^{2}}} x, m\right)\right],
\end{aligned}
$$

with the associated coupled dark soliton solution

$$
\begin{aligned}
& u=\frac{\sqrt{\beta}}{2}[1+\tanh (\sqrt{\beta} x)], \\
& v=-\frac{\sqrt{\beta}}{2}[1-\tanh (\sqrt{\beta} x)] .
\end{aligned}
$$

Figure 2 plots these solutions. Here too, $(-\mathfrak{u},-\boldsymbol{v})$ is also a solution. 

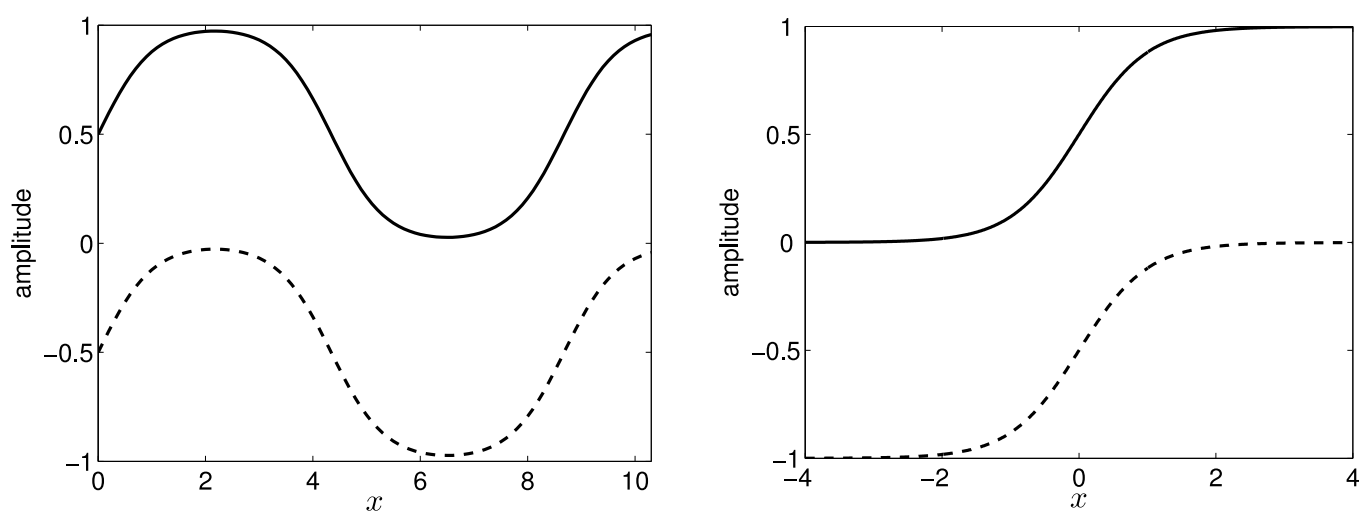

Figure 2: Exact in-phase solutions for $\beta=1$ and $\alpha=3$. To the left are the periodic solutions and to the right the soliton solutions with fields $u$ (solid line) and $v$.

We gain further insight into these solutions if we note that equations (4)-(5) describe the trajectory of a particle moving through the potential (for $\alpha=3$ )

$$
\Pi(u, v)=\beta\left(u^{2}+v^{2}\right)-\frac{1}{2}\left(u^{4}+v^{4}\right)-3 u^{2} v^{2},
$$

which has global maxima at $( \pm \sqrt{\beta}, 0)$ and $(0, \pm \sqrt{\beta})$ and saddle points $( \pm \sqrt{\beta} / 2, \pm \sqrt{\beta} / 2)$. Note that equations (4)-(5) can be normalised with respect to the parameter $\beta$ under the transformation of variables $z \rightarrow z / \beta^{1 / 2}$, $u \rightarrow u \beta^{1 / 2}$ and $v \rightarrow v \beta^{1 / 2}$. We therefore set $\beta=1$ for the purpose of numerical analysis but otherwise we keep it explicitly. Figure 3 plots the potential.

Firstly we show separatrix trajectories in $(u, v)$ space that correspond to known solutions. Defining the degree of ellipticity as $q(x)=(u-v) /(u+v)$, then trajectories in the $(u, v)$ plane that connect opposite maxima are the circularly polarised $(q= \pm 1)$ dark soliton solutions $( \pm \sqrt{\beta} \tanh (\sqrt{\beta} x), 0)$ and $(0, \pm \sqrt{\beta} \tanh (\sqrt{\beta} x))$. On the other hand, separatrix trajectories that connect opposite saddle points are the linearly polarised $(q=0)$ dark soliton 


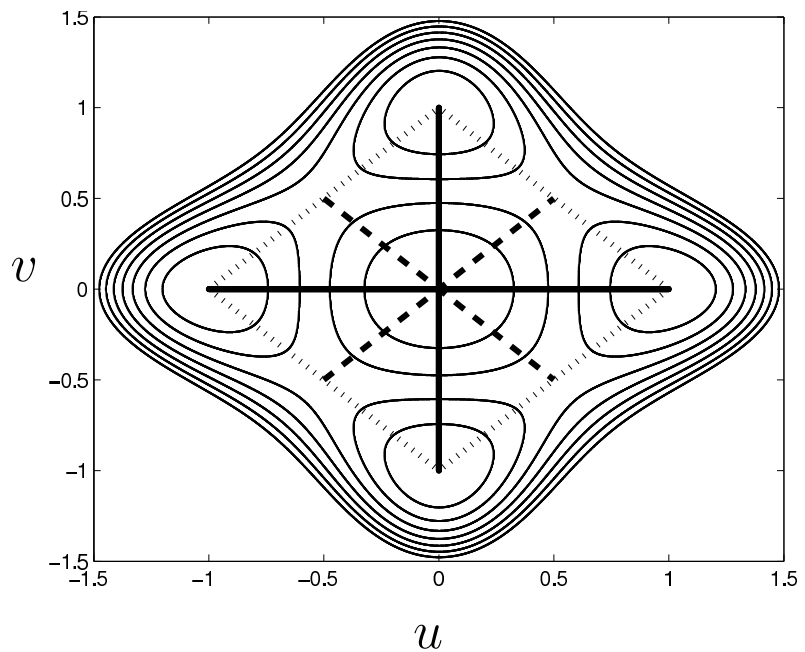

Figure 3: Contour plot of the potential function for $\beta=1$ and $\alpha=3$.

solutions $( \pm \sqrt{\beta} \tanh (\sqrt{\beta} x) / 2, \pm \sqrt{\beta} \tanh (\sqrt{\beta} x) / 2)[7,12]$. These constitute the well known (traditional) dark soliton solutions. Likewise we identify additional dark soliton solutions whose trajectory connects adjacent maxima. These are the solutions given above. The periodic solutions are obtained by starting at points on the separatrix other than at the maximum. Of particular interest are the solutions given by equations (10)-(11) as they form the boundary between regions of different polarisations and are called domain walls [12].

The obvious question is whether these solutions are physically relevant. The value of $\alpha=3$ is certainly outside the bounds of the usual values for $\alpha$, namely $2 / 3 \leqslant \alpha \leqslant 2$. We stress that soliton properties are dependent on the tensorial nature of the the third order nonlinear susceptibility whose values depend on the mechanism responsible for the induced nonlinearity. Haelterman and Sheppard [7] considered several different values of $\alpha$, particularly $\alpha=1.2,2,7,40$. The range $2 / 3 \leqslant \alpha \leqslant 2$ depicts vector solitons in different polarisation states where the nonlinearity is electronic in origin. In contrast, 
the nonlinearity induced by molecular reorientation corresponds to $\alpha \leqslant 7$ [4]. So our choice of $\alpha=3$ might indeed be both useful and relevant. Besides, the soliton solutions we developed are also significant from the mathematical point of view as they describe another complete set of vector soliton solutions for an important subset of coupled nonlinear evolution equations.

\section{Linear stability analysis}

To consider the stability of the dark soliton solution to equations (1)-(2) when $\alpha=3$ we assume a perturbation of the form

$$
\begin{aligned}
& \Psi_{1}=\left(u_{0}+u_{1} e^{\lambda z}\right) e^{i \beta z}, \\
& \Psi_{2}=\left(v_{0}+v_{1} e^{\lambda z}\right) e^{i \beta z},
\end{aligned}
$$

where $\mathfrak{u}_{0}=\mathfrak{u}_{0}(x)$ and $v_{0}=v_{0}(x)$ are the domain walls expressed by equations (10)-(11), $\mathfrak{u}_{1}=\mathfrak{u}_{1}(\mathrm{x})$ and $\boldsymbol{v}_{1}=v_{1}(\mathrm{x})$ are the complex perturbation eigenmodes $\left(\left|\mathfrak{u}_{1}\right| \ll \mathfrak{u}_{0},\left|v_{1}\right| \ll v_{0}\right)$, and $\lambda$ is the perturbation eigenvalue. Substituting equations (14)-(15) into equations (1)-(2) and keeping terms to first order in $u_{1}$ and $v_{1}$ we obtain the system of equations

$i \lambda u_{1}-\frac{1}{2} \frac{\partial^{2} u_{1}}{\partial x^{2}}-\beta u_{1}+u_{0}^{2}\left(u_{1}^{*}+u_{1}\right)+u_{1}\left(u_{0}^{2}+\alpha v_{0}^{2}\right)+\alpha u_{0} v_{0}\left(v_{1}+v_{1}^{*}\right)=0$, $i \lambda v_{1}-\frac{1}{2} \frac{\partial^{2} v_{1}}{\partial x^{2}}-\beta v_{1}+v_{0}^{2}\left(v_{1}^{*}+v_{1}\right)+v_{1}\left(v_{0}^{2}+\alpha u_{0}^{2}\right)+\alpha v_{0} u_{0}\left(u_{1}+u_{1}^{*}\right)=0$.

This system is expanded into a system of four linear equations by rewriting the eigenmodes so that the real and imaginary parts are explicit, namely, $u_{1}=u_{r}+i u_{i}$ and $v_{1}=v_{r}+i v_{i}$. This leads to solving an eigenvalue problem of the form

$$
\mathcal{L} X=\lambda X
$$

where $X=\left(u_{r}, u_{i}, v_{r}, v_{i}\right)^{\top}$ and $\mathcal{L}$ is a $4 \times 4$ matrix differential operator. If $\mathfrak{R}(\lambda)>0$, the perturbations grow without bounds with propagation distance $z$ and will destabilise the solitons. Using the ARPACK software package [13] the eigenvalues of largest magnitude and with largest real part were 
calculated. The numerics indicated that the real part of $\lambda$ was always zero to the limit of numerical accuracy $\left(10^{-16}\right)$. This indicates that the domain wall solitons are stable.

Next we propagated the domain wall soliton using a beam propagation method based on rational Chebyshev polynomials (RCP) as described by Towers and Jovanoski [14]. The boundary conditions employed are

$$
\begin{array}{rlrl}
\lim _{z \rightarrow-\infty}\left|\Psi_{1}\right| & =0, & & \lim _{z \rightarrow \infty}\left|\Psi_{1}\right|=1, \\
\lim _{z \rightarrow-\infty}\left|\Psi_{2}\right|=1, & & \lim _{z \rightarrow \infty}\left|\Psi_{2}\right|=0 .
\end{array}
$$

The advantage of using rational Chebyshev polynomials instead of the more traditional Fourier modes is that the latter uses periodic boundary conditions. This means that optical fields which propagate out of the calculation window re-enter the calculation window on the other side. This is an unphysical situation which can lead to unwanted interference effects. To rectify this an exponentially ramped dampening of the field towards the periphery of the numerical window was implemented. This method works well if the field amplitudes at the edge of the window are small but this is not the case for the Neumann boundary conditions given above. No such problems arise with the RCP method which is particularly suited to Neumann boundary conditions as discussed in detail by Towers and Jovanoski [14].

All calculations were performed to a relative error of less than $0.1 \%$. In each case we set $\beta=1$, and with an initial random perturbation of the field amplitude by $5 \%$ about the central core. Figure 4 plots the intensities $\left|\Psi_{1}\right|^{2}$ and $\left|\Psi_{2}\right|^{2}$ of the domain wall as it propagates through the medium up to $z=1000$. It is evident from the plots that the perturbations do not grow with propagation distance, consistent with the above linear stability analysis. The structural integrity of the domain wall soliton is maintained for large propagation distances leading us to conclude that the domain wall is stable. 



Figure 4: Stable propagation of domain wall solitons for $\beta=1$ and $\alpha=3$. Initial perturbation of $5 \%$ in the soliton amplitude.

\section{Conclusion}

We have found a new set of exact analytic solutions for a coupled system nonlinear Schrödinger equations in a defocussing material where the nonlinearity is induced by molecular reorientation. These solutions are found in terms of (periodic) Jacobi elliptic functions. As illustrated by phase plane analysis, these new periodic solutions reduce to dark solitary wave solutions which are either in-phase or out-of-phase in different parameter regimes.

Linear stability analysis and numerical simulations both indicate that domain wall solitons are stable. This implies that domain wall solitons should in principle be observable. Pitois et al. [15] reported the generation of domain wall structures in bimodal fiber. These are not the same as the domain wall solitons reported here but provide tantalising evidence for these possibly being observed and being manipulated for the purpose of optical data transmission devices. 


\section{References}

[1] Q. H. Park and H. J. Shin. Painlevé analysis of the coupled nonlinear Schrödinger equation for polarized optical waves in an isotropic medium. Phys. Rev. E 59, 1999, 2372-2379. doi:10.1103/PhysRevE.59.2373 C1053

[2] M. Segev. Optical spatial solitons. Opt. Quantum Electron. 30, 1998, 503-533. doi:10.10233/A:1006915021865 C1053

[3] G. P. Agrawal. Nonlinear Fiber Optics. 2nd edition. Academic Press, London, 1995. C1053

[4] Y. S. Kivshar and G. P. Agrawal. Optical Solitons: From Fibers to Photonic Crystals. Academic Press, London, 2003. C1053, C1060

[5] S. C. Tsang, K. Nakkeeran, B. A. Malomed and K. W. Chow. Coupled periodic waves with opposite dispersions in a nonlinear optical fiber. Opt. Comm. 249, 2005, 117-128. doi:10.1016/j.optcom.4224.12.042 C1054

[6] A. V. Porubov and D. F. Parker. Some general periodic solutions to coupled nonlinear Schrödinger equations. Wave Motion 29, 1999, 97-109. doi:10.1016/S0165-2125(98)00033-X C1054

[7] M. Haelterman and A. P. Sheppard. Extended modulation instability and a new type of solitary wave in coupled nonlinear Schrödinger equations. Phys. Lett. A 185, 1994, 265-272. doi:10.1016/0375-9601(94)90614-9 C1054, C1057, C1059

[8] F. T. Hioe. Periodic solitary waves for two coupled nonlinear Klein-Gordon and Schrödinger equations. J. Phys. A: Math. Gen. 36, 2003, 7307-7330. doi:10.1088/0305-4470/36/26/307 C1054 
[9] P. L. Christiansen, J. C. Eilbeck, V. Z. Enolskii and N. A. Kostov. Quasi-periodic solutions of the coupled Schrödinger equations. Proc. R. Soc. Lond. A 451, 1995, 685-700. doi:10.1098/rspa.1995.0149 C1054

[10] Z. Jovanoski, N. Ansari, I. N. Towers and R. A. Sammut. Exact domain-wall solitons. Phys. Lett. A 372, 2008, 610-612. doi:10.1016/j.physleta.2007.07.068 C1056

[11] C. Liu. Doubly periodic solutions of the coupled scalar field equations. Chaos, Solitons \& Fractals 23, 2005, 93-100. doi:10.1016/j.chaos.2004.03.022

[12] M. Haelterman and A. P. Sheppard. Vector solitons with polarization modulational instability in the normal-dispersion regime. Phys. Rev. E 49, 1994, 3389-3399.doi:10.1103/PhysRevE.49.3389 C1059

[13] http://www.caam.rice.edu/software/ARPACK/ C1060

[14] I. N. Towers and Z. Jovanoski. Application of rational Chebyshev polynomials to optical problems. ANZIAM J. 50, 2008, C60-C74. http://anziamj.austms .org.au/ojs/index.php/ANZIAMJ/article/ view/1396 C1061

[15] S. Pitois, G. Millot, P. Grelu and M. Haelterman. Generation of optical domain-wall structures from modulation instability in a bimodal fiber. Phys. Rev. E 60, 1999, 994-1000. doi:10.1103/PhysRevE.60.994 C1062

\section{Author addresses}

1. Z. Jovanoski, School of Physical, Environmental and Mathematical Sciences, University of New South Wales at Australian Defence Force Academy, Canberra, Australia. mailto:z.jovanoski@adfa.edu. au

2. I. N. Towers, School of Physical, Environmental and Mathematical 
Sciences, University of New South Wales at Australian Defence Force Academy, Canberra, Australia.

3. N. A. Ansari, School of Physical, Environmental and Mathematical Sciences, University of New South Wales at Australian Defence Force Academy, Canberra, Australia. 